\title{
APLICABILIDADE DO CHECKLIST DE CIRURGIA SEGURA EM CENTROS CIRÚRGICOS HOSPITALARES
}

\author{
Safe surgery checklist applicability in hospital surgery centers \\ Aplicabilidad de la lista de cirugía segura en centros quirúrgicos de los hospitales \\ Rayanne Morais de Souza', Maria Gabriella Silva Araújo², Regina Célia Sales Santos Veríssimoº, \\ Isabel Comassetto ${ }^{4}$, Fabiana Andrea Soares Ferreira ${ }^{5}$, Thaís Honório Lins Bernardo ${ }^{6}$
}

RESUMO: Objetivo: Identificar a aplicabilidade do checklist de cirurgia segura em centros cirúrgicos hospitalares. Método: Pesquisa quantitativa, realizada em nível nacional, após aprovação do Comitê de Ética em Pesquisa da Universidade Federal de Alagoas, via correio eletrônico, com enfermeiros associados à SOBECC e que atuam em Centro Cirúrgico. A análise dos dados foi realizada pelo teste do $\chi^{2}$. Resultados: Todos os participantes (100\%) conhecem o checklist. Dos 113 participantes da pesquisa que o aplicam, 89 (78,76\%) observaram mudanças na comunicação interpessoal da equipe cirúrgica e $94(83,18 \%)$ afirmaram que após a aplicação do checklist houve melhorias na atuação profissional na área assistencial. As principais facilidades para a aplicação do checklist foram o preenchimento rápido e fácil e a organização do serviço. A falta de participação da equipe foi a principal dificuldade referida pelos sujeitos da pesquisa. Conclusão: A aplicação do checklist de cirurgia segura contribui para a qualidade da assistência prestada ao paciente cirúrgico. Palavras-chave: Centro cirúrgico hospitalar. Lista de checagem. Segurança do paciente.

ABSTRACT: Objective: To identify the safe surgery checklist applicability in hospital surgery centers. Method: This is a quantitative research carried out at national level, after approval by the Research Ethics Committee of Universidade Federal de Alagoas. The research was performed using e-mail, and included nurses who were members of Associação Brasileira de Enfermeiros de Centro Cirúrgico, Recuperação Anestésica e Centro de Material e Esterilização (SOBECC) and worked in surgery centers. Data analysis was performed by means of the chi-square test. Results: All the participants (100\%) were aware of the checklist. Of the 113 research participants who used the checklist, 89 (78.76\%) participants observed changes in the surgical team's interpersonal communication, and 94 (83.18\%) participants confirmed that after the checklist implementation, there were improvements in professional assistance performance. The main advantages of the checklist implementation were rapid and easy completion and service organization. Team's disengagement was the main difficulty reported by the research subjects. Conclusion: The safe surgery checklist application contributes to the quality of care provided to surgical patients. Keywords: Surgery department, hospital. Checklist. Patient safety.

RESUMEN: Objetivo: Identificar la aplicabilidad de la lista de cirugía segura en centros quirúrgicos de los hospitales. Método: Estudio cuantitativo, llevado a cabo a nivel nacional, después de la aprobación del Comité de Ética en Investigación de la Universidad Federal de Alagoas, vía correo electrónico, con las enfermeras asociadas al CSSD (SOBECC) y que trabajan en un centro quirúrgico. El análisis de los datos se realizó mediante el test de $\chi 2$. Resultados: Todos los participantes (100\%) conoce la lista de comprobación. De los 113 participantes en el estudio que se aplican, 89 (78.76\%) observaron cambios en la comunicación interpersonal del equipo quirúrgico y 94 (83.18\%) informó de que, tras la aplicación de la lista de comprobación, ha habido mejoras en la práctica profesional en el área asistencial. Las principales facilidades para la aplicación de la lista de comprobación fueron el llenado rápido y sencillo, y la organización del servicio. La falta de participación del equipo fue la principal dificultad reportadas por los sujetos de la investigación. Conclusión: la aplicación de la lista de comprobación de cirugía segura contribuye a la calidad de la atención dada a los pacientes quirúrgicos.

Palabras clave: Servicio de cirurgía en hospital. Lista de verificación. Seguridad del paciente.

'Enfermeira pela Escola de Enfermagem e Farmácia da Universidade Federal de Alagoas (UFAL). E-mail: raymsouzaoliveira@gmail.com

2Mestranda em Enfermagem pelo Programa de Pós Graduação em Enfermagem da UFAL. E-mail: gabriellaaraujo2@hotmail.com

'Doutora em Biotecnologia pela Rede Nordeste de Biotecnologia (RENORBIO). Docente em Enfermagem da UFAL. E-mail: salesregina@hotmail.com

4Doutora em Ciências pela Universidade de São Paulo (USP). Docente em Enfermagem da UFAL. E-mail: isabelcomassetto@gmail.com

${ }_{5}^{5}$ Enfermeira. Mestre em Saúde do Adulto pela USP. Docente em Enfermagem da UFAL. E-mail: fabisoaresferreira@gmail.com

${ }^{6}$ Doutora em Biotecnologia pela RENORBIO. Docente em Enfermagem da UFAL. E-mail: thais.bernardo@esenfar.ufal.br

Avenida Lorival Melo Mota, s/n - Cidade Universitária, Tabuleiro dos Martins - CEP. 57072-900 - Maceió (AL), Brasil.

Recebido: 03 jul. 2016 - Aprovado: 14 set. 2016

DOI: $10.5327 / Z 1414-4425201600040003$ 


\section{INTRODUÇÃO}

Alguns erros cometidos pelos profissionais de saúde repercutem fortemente na vida dos pacientes. Os eventos adversos que mais ocorrem são a realização de cirurgias em pacientes trocados ou erros de lateralidade ${ }^{1}$.

A estratégia adotada pela Organização Mundial de Saúde (OMS) para alcançar a segurança do paciente cirúrgico foi a criação e implementação, nas instituições de saúde, de uma lista de verificações, um checklist padronizado, elaborado para ajudar as equipes cirúrgicas a reduzirem as ocorrências de danos aos pacientes ${ }^{2}$. Esse instrumento é direcionado para as medidas de segurança no intraoperatório, no entanto, os períodos pré- e pós-operatório também são de suma importância para a segurança do paciente cirúrgico ${ }^{3}$.

A lista de verificação caracteriza-se como um checklist padrão que deve ter a participação de toda a equipe cirúrgica - anestesista, cirurgião, assistentes e profissionais de enfermagem. É composto por três etapas: a primeira checagem (Sign In) acontece antes da indução anestésica, já com o paciente na sala de cirurgia. A segunda checagem (Time Out) é realizada antes da incisão cirúrgica, e a última checagem (Sign Out) ao final do procedimento e antes que o paciente deixe a sala de cirurgia em direção à sala de recuperação ${ }^{4}$.

A implementação do checklist é rápida e de baixo custo, e orienta-se que apenas uma pessoa seja responsável por essa aplicação. Apesar de o enfermeiro ser o profissional mais indicado para orientar a checagem, qualquer profissional que participa do procedimento cirúrgico pode ser o coordenador da verificação. Se necessário, esse profissional deve ter autoridade sobre o processo cirúrgico para interromper ou impedir seu avanço, visto que, muitas vezes, são os pequenos detalhes que passam despercebidos ${ }^{5}$.

Este estudo justifica-se pela necessidade de aprofundar as pesquisas sobre este tema, a fim de que as organizações hospitalares e os profissionais de saúde tenham mais conhecimento da importância de processos assistenciais seguros, tornando-se relevante por garantir excelência e qualidade no cuidado prestado ao paciente cirúrgico.

\section{OBJETIVO}

Identificar a aplicabilidade do checklist de cirurgia segura em Centros Cirúrgicos hospitalares.

\section{MÉTODOS}

Trata-se de uma pesquisa quantitativa com aprovação do Comitê de Ética em Pesquisa da Universidade Federal de Alagoas, CAAE: 42024315.9.0000.5013. Docentes e enfermeiros das cinco regiões do país, que atuam na assistência e no gerenciamento do serviço no centro cirúrgico e tiveram seus e-mails disponibilizados pela Sociedade Brasileira de Enfermeiros de Centro Cirúrgico, Recuperação Anestésica e Centro de Material e Esterilização (SOBECC), participaram do estudo. Para compor a amostra deste estudo, o critério de inclusão adotado foi: atuar como enfermeiro no setor de centro cirúrgico. Os critérios de exclusão foram atuar em Central de Material e Esterilização (CME) e Recuperação Pós Anestésica (RPA), bem como não estar associado à SOBECC.

A coleta foi realizada no período de setembro a novembro de 2015 e garantiu a confidencialidade e a privacidade das informações prestadas. O convite para participar da pesquisa foi enviado por e-mail, contendo as orientações e as justificativas do presente estudo, juntamente com o Termo de Consentimento Livre e Esclarecido (TCLE) e o questionário.

$\mathrm{O}$ instrumento de coleta de dados utilizado nesta pesquisa foi uma adaptação do questionário usado no estudo: "Checklist de cirurgia segura: análise da segurança e comunicação das equipes de um hospital escola", realizado no interior do estado de São Paulo5.

Os dados coletados foram tabulados e posteriormente tratados em software de análise estatística para uso em pesquisas - Statistical Package for Social Sciences (SPSS) 20 -, e a análise dos dados ocorreu pelo teste do $\chi^{2}$. O valor p menor que 0,05 foi considerado estatisticamente significante.

\section{RESULTADOS}

O estudo contou com a participação de 147 enfermeiros atuantes em todas as regiões do Brasil. A região Sudeste foi a que apresentou maior representatividade na amostra, com 67 respondentes $(45,57 \%)$, seguida pela região Sul, com a participação de $36(24,48 \%)$ sujeitos (Tabela 1). Vale ressaltar a importância da representação de todas as regiões do Brasil neste estudo, por retratar a aplicabilidade do checklist em nível nacional.

A faixa etária predominante entre os sujeitos da pesquisa foi de 30 a 39 anos representada por $60(40,82 \%)$ participantes, seguida da faixa etária 40 - 49 anos com 39 (26,53\%) participantes. Os sujeitos acima de 50 anos correspondem a $29(19,73 \%)$ indivíduos, seguidos 
por 19 participantes entre 22 e 29 anos (12,92\%). A distribuição entre gêneros foi dissimilar, com 132 (89,80\%) participantes do sexo feminino e $15(10,20 \%)$ do sexo masculino.

A Tabela 2 ilustra a predominância da especialização como nível de formação dos sujeitos da pesquisa - 78 (53,06\%) participantes do estudo. Em seguida, a participação dos enfermeiros com mestrado caracteriza 37 participantes $(25,18 \%)$.

O tempo de experiência profissional em centro cirúrgico demonstra que $37(25,17 \%)$ sujeitos da pesquisa têm experiência profissional igual ou superior a 16 anos, 31 (21,08\%) têm de 6 a 10 anos. Em seguida, os sujeitos com experiência profissional entre 3 e 5 anos ou 11 e 15 anos são representados por 27 (18,37\%) participantes. Em menor número, são descritos 25 profissionais com experiência de 0 a 2 anos (17,00\%).

Entre os 147 sujeitos da pesquisa, 138 (93,87\%) atuam na assistência ou na gerência em centro cirúrgico, 8 (5,44\%) são docentes de enfermagem na área estudada e apenas $1(0,68 \%)$ é residente de enfermagem.

Dentre os 147 sujeitos da pesquisa, 100,00\% conhecem o checklist de cirurgia segura; 113 (76,87\%) afirmaram que o checklist de cirurgia segura é aplicado no centro cirúrgico

Tabela 1. Distribuição geográfica dos sujeitos da pesquisa. Maceió, Alagoas. Brasil, 2015.

\begin{tabular}{|c|c|c|}
\hline Variável & $\mathbf{n}$ & $\%$ \\
\hline Distribuição geográfica dos participantes \\
\hline Região Norte & 8 & 5,44 \\
\hline Região Nordeste & 25 & 17,00 \\
\hline Região Sul & 36 & 24,49 \\
\hline Região Sudeste & 67 & 45,58 \\
\hline Região Centro-oeste & 11 & 7,49 \\
\hline Total & 147 & 100,00 \\
\hline
\end{tabular}

n: número de participantes; \%: frequência.

Tabela 2. Distribuição do nível de formação dos participantes do estudo. Maceió, Alagoas. Brasil, 2015.

\begin{tabular}{|c|c|c|}
\hline Variável & $\mathbf{n}$ & $\%$ \\
\hline Nível de formação & & \\
\hline Graduação & 13 & 8,84 \\
\hline Especialização & 78 & 53,06 \\
\hline Mestrado & 37 & 25,18 \\
\hline Doutorado & 18 & 12,24 \\
\hline Pós-doutorado & 01 & 0,68 \\
\hline Total & 147 & 100,00 \\
\hline
\end{tabular}

n: número de participantes; \%: frequência. em que trabalham; e $34(23,12 \%)$ não aplicam o checklist. Todos os $34(23,12 \%)$ sujeitos que não aplicam o checklist relataram que gostariam que o mesmo fosse aplicado caso estivessem atuando como enfermeiros em uma sala cirúrgica.

Verificou-se predominância da aplicação do checklist nos serviços de saúde de iniciativa privada - 47 (41,59\%) participantes - , porém a discrepância em relação à rede pública $42(37,16 \%)$ indivíduos — não foi acentuada. Os sujeitos da pesquisa que atuam concomitantemente nas redes pública e privada representam $24(21,23 \%)$ dos participantes.

Dos 113 sujeitos da pesquisa que aplicam o checklist no centro cirúrgico em que trabalham, 89 (78,76\%) afirmaram que a aplicação provocou mudanças na comunicação interpessoal da equipe cirúrgica e $24(21,24 \%)$ não observaram essas mudanças. Ao relacionar as mudanças na comunicação interpessoal com a rede pública ou privada dos estabelecimentos de saúde, houve diferença estatística significativa ( $\mathrm{p}=0,013$ ), visto que, dentre os 47 sujeitos que atuam na rede privada, $42(89,4 \%)$ observaram mudanças na comunicação e apenas 5 (10,6\%) não observaram essas mudanças (Tabela 3). Pode-se constatar que as mudanças na comunicação interpessoal da equipe cirúrgica a partir da aplicação do checklist foram observadas com maior frequência pelos sujeitos da pesquisa que atuam nos serviços de saúde da iniciativa privada.

Ao elencar as dificuldades e facilidades para a aplicação do checklist de cirurgia segura, os sujeitos da pesquisa puderam referir mais de uma opção. Dentre os 113 sujeitos que aplicam o checklist no centro cirúrgico em que atuam, 59 (52,21\%) citaram preenchimento rápido e fácil e 44 (38,94\%) mencionaram organização do serviço, sendo essas as facilidades citadas com maior frequência, seguidas por baixo custo $42(37,17 \%)$ - e agilidade na assistência $22(19,46 \%)$.

O caráter do estabelecimento de saúde (público ou privado) também apresentou diferença estatística, quando relacionado à agilidade na assistência $(\mathrm{p}=0,006)$ (Tabela 4). Dos

Tabela 3. Mudanças na comunicação interpessoal da equipe cirúrgica a partir da aplicação do checklist. Maceió, Alagoas. Brasil, 2015.

\begin{tabular}{|l|c|c|c|c|c|}
\hline \multirow{2}{*}{ Variável } & \multicolumn{2}{|c|}{ Sim } & \multicolumn{2}{|c|}{ Não } & \multirow{2}{*}{ Valor p } \\
\cline { 2 - 5 } & $\mathbf{n}$ & $\%$ & $\mathbf{n}$ & $\%$ & \\
\hline Estabelecimento de saúde em que trabalha \\
\cline { 1 - 4 } \\
\cline { 1 - 4 } Pública & 27 & 64,3 & 15 & 35,7 & \\
\hline Privada & 42 & 89,4 & 5 & 10,6 & 0,013 \\
\hline Ambos & 20 & 83,3 & 4 & 16,7 & \\
\hline
\end{tabular}

$n$ : número de participantes; \%: frequência; $p<0,05$ : diferença estatística significativa. 
113 sujeitos da pesquisa que aplicam o checklist, 91 (80,53\%) não referem agilidade na assistência como uma facilidade para a aplicação. Desses 91 indivíduos, 42 (46,15\%) atuam na rede privada, enquanto $35(38,47 \%)$ trabalham na rede pública.

Houve diferença estatística $(\mathrm{p}=0,003)$ significativa para o preenchimento rápido e fácil, de acordo com os estabelecimentos de saúde das redes pública e privada. Dos 113 sujeitos da pesquisa que aplicam o checklist no centro cirúrgico em que trabalham, $54(47,79 \%)$ afirmaram que o preenchimento é rápido e fácil. Dentre eles, 30 (55,55\%) atuam nos serviços de saúde da rede pública.

As opções propostas para dificuldades na aplicação do checklist foram: falta de participação da equipe, itens de difícil compreensão, falta de explicação sobre o checklist, preenchimento muito longo, não houve dificuldades para aplicação, entre outras. Neste caso, a falta de participação da equipe apareceu como a principal dificuldade, sendo referida por $88(77,88 \%)$ dos 113 sujeitos que aplicam o checklist no Centro Cirúrgico (CC) em que trabalham.

A Tabela 5 retrata que, face à formação profissional dos sujeitos da pesquisa relacionada às melhorias na atuação assistencial

Tabela 4. Agilidade na assistência como facilidade para a aplicação do checklist na rede pública e/ou privada. Maceió, Alagoas. Brasil, 2015.

\begin{tabular}{|c|c|c|c|c|c|}
\hline \multirow{2}{*}{ Variável } & \multicolumn{2}{|c|}{ Sim } & \multicolumn{2}{|c|}{ Não } & \multirow{2}{*}{ Valor p } \\
\cline { 2 - 5 } & $\mathbf{n}$ & $\%$ & $\mathbf{n}$ & $\%$ & \\
\hline Estabelecimento de saúde em que trabalha \\
\cline { 1 - 4 } \\
\cline { 1 - 4 } Pública & 7 & 16,7 & 35 & 83,3 & \\
\hline Privada & 5 & 10,6 & 42 & 89,4 & 0,006 \\
\hline Ambos & 10 & 41,7 & 14 & 58,3 & \\
\hline
\end{tabular}

n: número de participantes; \%: frequência; $p<0,05$ : diferença estatística.

Tabela 5. Melhorias para a atuação do enfermeiro no centro cirúrgico, na área assistencial, após a aplicação do checklist, de acordo com a formação profissional dos sujeitos da pesquisa. Maceió, Alagoas. Brasil, 2015.

\begin{tabular}{|l|c|c|c|c|c|}
\hline \multirow{2}{*}{ Variável } & \multicolumn{2}{|c|}{ Sim } & \multicolumn{2}{c|}{ Não } & \multirow{2}{*}{ Valor p } \\
\cline { 2 - 5 } & $\mathbf{n}$ & $\%$ & $\mathbf{n}$ & $\%$ & \\
\hline Formação Profissional & & & & & \\
\hline Graduação & 10 & 100 & 0 & 0,0 & \\
\hline Especialização & 46 & 75,4 & 15 & 7,4 & \multirow{2}{*}{0,016} \\
\hline Mestrado & 25 & 92,6 & 2 & 7,1 & \multirow{2}{*}{ Doutorado } \\
\hline Pós-doutorado & 13 & 92,9 & 1 & 100,0 & \\
\hline
\end{tabular}

n: número de participantes; \%: frequência; $p<0,05$ : diferença estatística. do enfermeiro após a aplicação do checklist, verificou-se diferença estatística significativa $(\mathrm{p}=0,016)$, com $94(83,19 \%)$ dos participantes afirmando que houve melhorias na atuação profissional na área assistencial e 19 (16,81\%) declarando que não houve melhorias. Desses 94 indivíduos, os profissionais com especialização - $46(48,93 \%)$ - e com mestrado - 25 (26,60\%) aparecem com maior frequência entre os sujeitos que referiram melhorias na assistência de enfermagem.

Do total de participantes, 91 (80,53\%) afirmaram que o checklist não contribuiu para a agilidade na assistência ao paciente cirúrgico. Para os sujeitos com experiência profissional superior a seis anos, a compreensão dos itens do checklist não foi uma dificuldade para sua aplicação, visto que apenas $7(6,20 \%)$ dos 113 participantes encontraram tal dificuldade. As regiões com maior aplicação deste instrumento foram Sudeste e Sul, representadas por 55 (48,68\%) e $27(23,90 \%)$, respectivamente.

\section{DISCUSSÃO}

É comum na maioria dos hospitais públicos brasileiros a presença de profissionais sobrecarregados, com baixos salários, em condições inadequadas de trabalho e desprovidos de protocolos de segurança, o que certamente aumenta a probabilidade de erros ${ }^{6}$.

A otimização da segurança do paciente cirúrgico deve ser implementada por todas as instituições de saúde, públicas e privadas, por meio de treinamentos e palestras sobre sua importância para os pacientes e profissionais de saúde. Quanto mais seguro for um procedimento cirúrgico, melhor será a qualidade da assistência, da segurança e da recuperação, tanto do paciente quanto da equipe multidisciplinar, ainda que muitos membros de equipes que trabalham em hospitais públicos e privados resistam à implantação do checklist, confiando em sua memória, sem levar em conta o cansaço decorrente de muitas horas de serviço ${ }^{6}$.

É preciso utilizar a checagem como meio de comunicação interpessoal, ou seja, como facilitador na assistência ao paciente. O uso do checklist contribui para diminuir os atritos provocados por situações inesperadas, e a apresentação dos membros da equipeantes do procedimento - melhora a segurança para o paciente cirúrgico ${ }^{5}$. Pode-se inferir que, com o checklist, a comunicação com toda a equipe acontece enquanto a mesma confirma itens e informa suas ações e preocupações a todos da sala de cirurgia.

Quando a comunicação entre a equipe de saúde não é efetiva, eventos como a suspensão de cirurgias, de procedimentos e de 
exames acabam se tornando comuns. Além disso, os pacientes ficam submetidos a longos períodos sem alimentação e, muitas vezes, não recebem a dieta adequada devido a essas falhas, que geram atrasos e não atendimento ao paciente ${ }^{1}$.

Muitos erros gerados por falhas no processo de comunicação podem ser irreversíveis. Os processos de comunicação são muito complexos e dinâmicos nos serviços de saúde. O alto fluxo de informações e o grande número de profissionais de diferentes equipes assistenciais, além da grande demanda de atividades, acarretam a necessidade de constante atualização e troca de informações com os pacientes, os familiares e as equipes. A falta de processos de comunicação integrados entre as diversas equipes de profissionais e os serviços de saúde é um fator que contribui para as falhas no atendimento ${ }^{1}$.

Nesse contexto, vale ressaltar que a equipe cirúrgica é composta por cirurgiões, anestesiologistas, equipe de enfermagem, técnicos e outras pessoas da sala de operações envolvidas na cirurgia. O recurso mais crítico para o sucesso do processo cirúrgico é a própria equipe. Desse modo, uma equipe que trabalha efetivamente unida para usar seus conhecimentos e habilidades em benefício do paciente cirúrgico pode prevenir uma proporção considerável das complicações que ameaçam sua vida ${ }^{1}$. Mais do que o preenchimento do checklist, os profissionais envolvidos no ato anestésico-cirúrgico devem resgatar as origens de sua formação humanística e ética ${ }^{7}$.

A principal dificuldade relatada neste estudo para a aplicação do checklist foi a falta de participação da equipe cirúrgica, o que comprova que o bom uso desse instrumento se dá quando os profissionais compreendem sua importância, fazendo-se necessária a participação de todos ${ }^{8}$. Ações educativas direcionadas à quebra de paradigmas - como a hierarquia do cirurgião - são uma estratégia para evitar problemas relacionados à aplicação do checklist e à falta de comprometimento da equipe cirúrgica ${ }^{9}$. Os esforços de gestores e profissionais devem ter como objetivo a consciência universal e o conhecimento completo sobre a importância e o uso correto do checklist de cirurgia segura, a fim de garantir a segurança do paciente e da equipe cirúrgica ${ }^{10}$.

Assim, para implantar o programa Cirurgias Seguras Salvam Vidas - da OMS - em uma organização de saúde que oferece assistência cirúrgica, deve-se fazer muito mais do que apenas aplicar uma lista de verificação de fluxo e das etapas do procedimento anestésico-cirúrgico. É imprescindível promover uma mudança na cultura de segurança do paciente para que todos os profissionais da equipe cirúrgica e da gestão da organização compreendam a necessidade e os benefícios desse protocolo para todas as pessoas envolvidas ${ }^{11}$.
O preenchimento rápido e fácil foi a característica relatada com maior frequência pelos participantes deste estudo. Estima-se que é necessário o tempo total de três minutos para a aplicação das três fases do processo de verificação do checklist, e orientase que uma única pessoa seja responsável por essa aplicação ${ }^{5}$.

O enfermeiro é o profissional mais indicado para orientar a checagem, porém qualquer profissional que participe do procedimento cirúrgico pode ser o coordenador da verificação. Diante dos resultados apresentados, pode-se inferir que o enfermeiro tornou-se mais participativo e atuante na sala operatória.

O checklist tem a intenção de fornecer um conjunto eficiente e simples de verificações prioritárias para promover o trabalho e a comunicação efetiva entre a equipe. O objetivo do checklist não é pronunciar algo decorado ou impedir o fluxo de trabalho. Desse modo, para introduzir a lista de verificação na sala de operação e para que as equipes aprendam a usá-la de forma efetiva, faz-se necessário colocá-la em prática ${ }^{4}$.

Retratar a aplicabilidade do checklist nas diversas regiões do país, em hospitais públicos e/ou privados, colabora para o entendimento dos desafios do processo de sua implantação. Vale ressaltar a importância de uma mudança na cultura organizacional dos gestores e profissionais de saúde, no sentido de compreenderem a segurança do paciente como essencial para a prevenção de eventos adversos.

Este estudo apresentou limitações importantes quanto à sua população e amostra, pois a coleta de dados foi realizada por email. Os resultados limitam-se à amostra investigada de enfermeiros associados à SOBECC, não permitindo generalizações à população total de enfermeiros que atuam em Centros Cirúrgicos de todo o país.

\section{CONCLUSÃO}

Todos os sujeitos participantes da pesquisa conhecem o checklist de cirurgia segura, que é aplicado com mais frequência nas regiões Sudeste e Sul e nos serviços de saúde da rede privada.

A aplicação do checklist provocou mudanças na comunicação interpessoal da equipe cirúrgica e melhorias na atuação do enfermeiro na área assistencial.

As facilidades encontradas para a aplicação do checklist foram o preenchimento rápido e fácil, a organização do serviço, e a agilidade na assistência. A falta de participação da equipe apareceu como a principal dificuldade para a aplicação do checklist, seguida de itens de difícil compreensão, preenchimento muito longo e falta de explicação sobre o checklist. 
Apesar de todos os participantes deste estudo conhecerem o checklist, isso não significa que sabem utilizá-lo corretamente. É imprescindível realizar treinamentos com todos os profissionais que atuarão na sala operatória a fim de sensibilizá-los sobre a importância e o uso correto desse instrumento.
Assim, faz-se necessário o aprimoramento do trabalho em equipe, visto que a aplicação do checklist de cirurgia segura tem o intuito de promover a segurança do paciente cirúrgico, proporcionando um ambiente seguro e comunicação interpessoal eficiente entre os componentes da equipe cirúrgica.

\section{REFERÊNCIAS}

1. Pires MPO, Pedreira MLG, Peterlini MAS. Surgical Safety in Pediatrics: practical application of the Pediatric Surgical Safety Checklist. Rev Latino-Am Enfermagem. 2015;23(6):1 105-12. [acesso em 2016 mar. 27]. Disponível em: http://www.scielo.br/scielo. php?script=sci_arttext\&pid=S0104-11692015000601105

2. Organização Mundial de Saúde. Segundo desafio global para a segurança do paciente: Cirurgias seguras salvam vidas; Ministério da Saúde; Agência Nacional de Vigilância Sanitária; 1a. ed. Brasília, DF; 2009 [acesso em 2016 mar. 29]. Disponível em: http://bvsms. saude.gov.br/bvs/publicacoes/seguranca_paciente_cirurgia_salva_ manual.pdf

3. Agência Nacional de Vigilância Sanitária. Assistência Segura: Uma reflexão teórica aplicada à prática. 1a. ed. Brasília, DF; 2013. [acesso em 2016 jan. 15]. Disponível em: http://www20.anvisa. gov.br/segurancadopaciente/images/documentos/livros/Livro1Assistencia_Segura.pdf

4. Salles CLS, Carrara D, Kusahara DM. Boas práticas - Cirurgia segura. Conselho Regional de Enfermagem de São Paulo. São Paulo; 2011. [acesso em 2016 fev. 11]. Disponivel em: http://inter.coren-sp.gov. $\mathrm{br} / \mathrm{sites} /$ default/files/cirurgia-segura.pdf

5. Pancieri AP, Avila MAG, Braga EM. Safe surgery checklist: analysis of the safety and communication of teams from a teaching hospital. Rev Gaúcha Enferm. 2013;34(1):71-8. [acesso em 2015 dez. 03]. Disponível em: http://www.scielo.br/scielo. php?script=sci_arttext\&pid=S1983-14472013000100009\&lng= en\&nrm=iso\&tlng=en
6. Porto KLH. A segurança do paciente na utilização do checklist. Rev Enf. 2014;17(2). [acesso em 2016 mar. 28]. Disponível em: http://periodicos.pucminas.br/index.php/enfermagemrevista/article/ view/8336/7195

7. Grazziano ES. Segurança no cuidado em cirurgias: onde estamos? Rev SOBECC. 2015;20(2):63. [acesso em 2016 mar. 28]. Disponível em: http://files.bvs.br/upload/S/1414-4425/2015/v20n2/a5015.pdf

8. Pancieri AP, Carvalho R, Braga EM. Aplicação do checklist para cirurgia segura: Relato de experiência. Rev SOBECC. 2014;19(1):26-33. [acesso em 2015 dez. 03]. Disponível em: http://sobecc.org.br/arquivos/ artigos/2015/pdfs/site_sobecc_v19n1/05_sobecc_v19n1.pdf

9. Maziero ECS, Silva AEBC, Mantovani MS, Cruz EDA. Adherence to the use of the surgical checklist for patient safety. Rev Gaúcha Enferm. 2015;36(4): 14-20. [acesso em 2016 jun. 24]. Disponível em: http://seer.ufrgs.br/index.php/RevistaGauchadeEnfermagem/article/ view/53716/35652

10. Hurtado JJD, Jiménez X, Peñalonzo MA, Villatoro C, Izquierdo S, Cifuentes M. Acceptance of the WHO Surgical Safety Checklist among surgical personnel in hospitals in Guatemala city. BMC Health Serv Re. 2012;12:169. [acesso em 2016 jun. 24]. Disponível em: http:// www.ncbi.nlm.nih.gov/pmc/articles/PMC3444374/

11. Corona ARP, Peniche ACG. A cultura de segurança do paciente na adesão ao protocolo de cirurgia segura. Rev SOBECC. 2015;20(3):179185. [acesso em 2016 mar. 29]. Disponível em: http://files.bvs.br/ upload/S/1414-4425/2015/v20n3/a5210.pdf 